\title{
Using Video Games to Regulate Emotions
}

\author{
ZHANNA SARSENBAYEVA, The University of Melbourne, Australia \\ BENJAMIN TAG, The University of Melbourne, Australia \\ SHU YAN, The University of Melbourne, Australia \\ VASSILIS KOSTAKOS, The University of Melbourne, Australia \\ JORGE GONCALVES, The University of Melbourne, Australia
}

In this paper we investigate the practice of using video games for digital emotion regulation. In a two-week diary study we collected participants' records on their emotional states and video games experience. Our findings show that people use video games to respond to their reality, to socialise and communicate with their friends. We also show that video games lead to intentional and unintentional emotional outcomes depending on different aspects of the game. Our work provides insights on using video games for digital emotion regulation and directions for future research.

CCS Concepts: • Human-centered computing $\rightarrow$ Empirical studies in HCI.

Additional Key Words and Phrases: Emotions, emotional well-being, digital emotion regulation, video games, smartphones

\section{ACM Reference Format:}

Zhanna Sarsenbayeva, Benjamin Tag, Shu Yan, Vassilis Kostakos, and Jorge Goncalves. 2020. Using Video Games to Regulate Emotions. In 32ND AUSTRALIAN CONFERENCE ON HUMAN-COMPUTER INTERACTION (OzCHI '20), December 2-4, 2020, Sydney, NSW, Australia. ACM, New York, NY, USA, 8 pages. https://doi.org/10. $1145 / 3441000.3441035$

\section{INTRODUCTION}

Emotions are an integral part of human behaviour and occur as a set of behavioural and physiological responses to challenges that people experience [12]. Negative emotions have been shown to have adverse impact on human physical and mental health associated with cardiovascular diseases [39] and mental disorders [6]. Meanwhile, positive emotions have been shown to improve educational performance [23] and the ability to concentrate [29]. Therefore, emotions play an important role in human well-being and the ability to regulate emotions can lead to improvements in people's mental and physical health [22].

The term "emotion regulation" implies that there exists a process separate from emotions used to modulate emotions [6] and generally refers to the process of modulating one or more aspects of an emotional experience or response [14]. Emotion regulation has been shown to act as an effective response for borderline personality disorders [25] as well as substance abuse, eating disorders, and depression [24]. Moreover, successful emotion regulation has been shown to reduce the risk of heart attacks and coronary heart disease [22].

People often use intentional strategies to regulate their emotions. Some tend to do sports, spend time with their loved ones, or interact with pets as it has been shown to improve mood and

Permission to make digital or hard copies of part or all of this work for personal or classroom use is granted without fee provided that copies are not made or distributed for profit or commercial advantage and that copies bear this notice and the full citation on the first page. Copyrights for third-party components of this work must be honored. For all other uses, contact the owner/author(s).

OzCHI '20, December 2-4, 2020, Sydney, NSW, Australia

(C) 2020 Copyright held by the owner/author(s).

ACM ISBN 978-1-4503-8975-4/20/12.

https://doi.org/10.1145/3441000.3441035 
emotional well-being [27]. Due to the current prevalence of digital technology, people also tend to default to digital technologies to regulate their emotions [47].

One example of such technology is video games. The popularity of video games has consistently grown over the past few years. In the US alone there are 155 million video game players $[3,20]$. Research has shown that video games lead players to experiencing a wide range of emotions, both positive and negative [20]. Therefore, understanding emotions produced by video games and their effect on emotional health provides new insights and opportunities for research in emotion regulation [20].

In this work, we investigate how people attempt to regulate their emotions by playing video games. We conducted a two-week diary study with 10 participants and found that participants play video games in order to regulate their emotions to respond to real-world situations. We also found that video games lead to both intentional and unintentional outcomes often depending on factors such as game result and game setting. We discuss potential benefits and risks of using video games for emotion regulation and outline opportunities for future work.

\section{RELATED WORK}

Researchers suggest that in order to promote mental health, it is essential to better conceptualise and, therefore, enable proactive emotion regulation [6,44]. According to Gross [15], it is important to understand that it is not the situation, but the individual's judgement of the situation that generates the emotion. Therefore, emotion regulation can be performed for example by changing the way of evaluating the situation (i.e., use an emotion regulation strategy) [15].

\subsection{Emotion Regulation Strategies}

In Psychology research there exists two main emotion regulation strategies: antecedent-focused and response-focused strategies [6]. Antecedent-focused strategies concentrate on the modification of the input to the emotion-generative system and include such manipulations as selecting/modifying the situation, monitoring attentional deployment, and cognitive reassessment of the situation to change the emotional salience $[4,6]$. Meanwhile, response-focused strategies work on altering the output of the emotion-generative system, e.g., by suppressing emotional expressions when emotionally aroused [6].

Nevertheless, inhibiting emotions has been shown to adversely affect emotional well-being [13]. For example, emotion inhibition increases the intensity and frequency of the cardiovascular and sympathetic system highly correlating with immune and cardiovascular problems $[13,34]$. It also impairs memory recall [34] and causes higher incidences of depression [18]. Cognitive reappraisal, on the other hand, has been shown to be effective in down-regulating negative emotions [16] and to have several benefits [6]. For instance, reappraisal is effective in reducing emotional experiences of anger and provides an improved sense of meaning without any physiological cost $[1,10]$. Moreover, it has been shown that cognitive reappraisal is more adaptive than the suppression of emotions [16].

Another widely adopted emotion regulation strategy is mindfulness. In contrast to reappraisal, mindfulness suggests that emotions are mental events and do not need to be acted upon [6]. Instead, mindfulness suggests developing a capacity to allow these mental events to come and go with a purpose to consciously choose behaviours and thoughts that lead to pleasant results [6]. Therefore, it is important to understand how people regulate their emotions as different emotion regulation strategies may have different consequences on an individual's well-being [17]. 


\subsection{Digital Media and Human Emotions}

There has been a recent emergence of interest in digital emotion regulation in Human-Computer Interaction (HCI) as digital technology has been shown to be successfully used for emotion regulation [47]. Different types of digital technologies have been shown to affect human emotional states [47].

For example, digitally-mediated content is being used to cope with stress, to induce desired emotions, and to distract from problems, by deflecting attention [46]. Furthermore, as consumers of digital media typically respond to mediated content as if it occurs in real life [11,32], it has been suggested to improve emotional well-being, thus, potentially being useful as a low-cost method to regulate emotions [2]. For example, online cat videos have been shown to generate pleasure and positive affect [28]. However, digital media can also cause negative emotions in people. For example, research has shown that individuals find spending time on online media as a way to procrastinate, potentially leading to increased feelings of guilt [33].

Moreover, recent research has shown that smartphone usage may influence users' emotional states as well as emotions might define users' smartphone usage [26, 36, 45]. Researchers also demonstrate that depending on the content of mobile applications and smartphone use behaviour [37], users may experience either positive or negative affect [36] and vice versa [42].

Another way to regulate emotions is to play video games due to the highly emotional nature of modern videos games [20]. Ravaja et al. [31] found that positive valence arousal was connected to active player engagement when playing video games. Collins and Cox [7] have demonstrated that video games can potentially be associated with positive recovery from work-related strain. Video games have also shown to cause aggressive emotions and frustration in players resulting from inadequate and hindered performance [30]. Moreover, there is some evidence of video games leading to emotional desensitisation to on-screen violence [5] and the inability to recognise emotions in others [21]. As can be seen from the above examples, the impact of video games on human emotional and mental states remains a subject for debate $[9,20]$. To contribute to this discourse, we are presenting our observations of participants' motivation to play video games and investigate how people use video games to regulate their emotions.

\section{METHOD}

We conducted a two-week diary study with 10 participants (5 men and 5 women) recruited via university mailing lists and through snowball recruitment. Participants' age varied between 18 and 35 years and they all reported to play video games on a regular basis (average game play time is 1.6 hours per day). The most commonly played games among our participants were "World of Warcraft", "Dota 2", "CSGO", "Animal Crossing: New Horizons", "Arena of Valor" and other less commonly played games such as "Candy Crush", "Angry Birds", "Civilization V", and "The Witcher 3”. Participants had different educational backgrounds (e.g., Biomedicine, Computer Science, Education, Literature) and original backgrounds (e.g., Australia, China, India, Indonesia, Saudi Arabia). Prior to enrolling in the study, all participants received instructions to observe and keep record of their daily video games experiences as well as emotional states before and after playing the game. To confirm their agreement to participate in the study, all participants provided written consent to the researchers. We sent our participants daily reminders to ensure they have kept a record of their emotional experience and experience of playing video games. We also provided our participants with a diary template and questions to support the data collection process. The participants also had the opportunity to enter open-ended comments when they felt it was necessary. At the end of the study we conducted online semi-structured face-to-face interviews with each participant to hear their reflections on the study and their experience. 


\section{RESULTS}

We conducted a thematic analysis on the collected qualitative data. Two of the authors performed initial coding of the data, after reading through each of the responses. Then the researchers performed a comparison of the initial codes and produced the final codes based on their similarity (e.g., "I play games to build up my confidence" and "I play games for fun" merged into "Intrinsic motivation to play video games"). The data was then again coded according to the final codes, which we then reviewed and finalised as themes presented below.

\subsection{Theme 1 - People sometimes play video games to respond to their every day life}

Participants stated that playing video games helps them to respond to reality. According to their diary records, different external and internal factors cause negative emotions and unpleasant feelings which they tend to regulate by playing video games. The majority of participants mentions that they play video games in order to respond to stress caused by external factors such as pressure of performing well at work and at university as well as dealing with deadlines. "This is my first year on the job and I'm busy working during the day to learn job-related skills. I was stressed out, so I play two hours of competitive games at night to relieve the pressure." (P03), "I am busy studying every day. I have too much homework to do. I feel anxious because of the heavy work, and I often play some casual games to relieve my study pressure" (P05).

Moreover, participants state that they play video games to respond to their unpleasant internal states in order to change their feelings, such as boredom and tiredness: "I think sometimes I'll play games to release my stress. But for most of time, I choose to play video games because I'm bored." (P01), "When I am in a bad mood or even angry, I choose to play some casual games to calm myself down. In the process of playing games, I slowly release the negative emotions." (P04).

Finally, participants reported that video games help them to immerse with the virtual world,and thereby, escape reality. For example, several participants mentioned that the video games they play let them feel things that do not exist in their everyday life: "A lot of the rules of the real world have been made and I can't change them. But in the game [it] is not the same, I can be a top team's head coach, can be a dragon warrior, can be an excellent soldier driving the mech. I will play some simulation games and experience things that I can't experience in real life. The pressure in real life is released in virtual online games" (P08). This was particularly important for participants with children as video games help them relax after a day full of childcare responsibilities. "My baby was grumpy today, I needed to do something to relax after he [fell] asleep" (P01), "Playing games helps me escape real life and change my emotions. Otherwise, I'm going crazy about taking care of my child." (P10).

\subsection{Theme 2 - Video games are means of socialisation}

We also found that our participants considered video games as means of socialising with their friends. Several participants mentioned they play video games to catch up with friends and to spend good time together: "It feels good to play games with friends" (P03), "Playing games with friends makes me feel less stressed" (P08), "After a work day, I play video games with my friends to chat and win some games to gain confidence and release the pressure" (P09).

Furthermore, participants recorded that the setting of the game plays a significant role in helping them to regulate their emotions. For example, jokes and funny moments that occurred during video games sessions with friends brought positive emotions to our participants: "Friend's jokes made me happy" (P02), "Happy with my friend's joke and relaxed" (P04), "My friend's bad performance made me laugh at him and made me very happy" (P10). 


\subsection{Theme 3 - Video games lead to intentional and unintentional emotional outcomes}

We found that after playing games our participants felt either positive and negative emotions depending on different factors. For example, participants reported feeling negative emotions because they lost or were outperformed by their competitors: "I lost! I was so upset" (P02), "After losing [the game] not in a good mood" (P03). Meanwhile, participants felt positive emotions when they won: [I felt] happier after winning the game (P02), outperformed their competitors: "Because I surpassed my colleagues, I was very excited" (P01) or reached some achievement: "I am happy because I passed the level" (P01), "New top score makes me feel happy" (P04). The above examples describe how the result of the game led to unintentional emotions of our participants. Some participants claim that this then helps them improve their performance at work and at school: "I like playing competitive games. Every victory inspires me. I enjoy the feeling of success. By playing competitive games I constantly refresh my ranking, which makes my blood boil. I can continue this positive state into my study." (P07),

Not only the result of the game, but the game setting itself influenced participants' emotional states - without the players' intent - both negatively and positively. By definition, this would not qualify as emotion regulation, as emotion regulation requires a person's intent to modify emotions $[12,15,19]$. However, as all our candidates are regular players, they are aware of the potential of surprising events happening in a game, we report these unintentional instances as well. For example, one participant reported feeling "annoyed because of broken game functions" (P10) while another participant reported feeling happy "because of getting a good equipment" (P08). Furthermore, participants reported feeling guilty and frustrated when they played for long time, as they felt they have "wasted" their time: "Frustrated because of playing for too long and slept so late" (P04), "Playing games for too long makes me feel guilty" (P07), "Playing late makes me a little worried about tomorrow's work" (P09).

However, we also found that participants played video games with the clear intention of experiencing positive emotions, particularly due to game characteristics. For instance, several participants mentioned that the game scenery and music lead them to feeling positive affect: "The beautiful game scenery makes me relaxed" (P03), "Attracted by the game world, I am very happy to be immersed in the beautiful scenery" (P07), "I feel free and relaxed in the game world" (P10).

\section{DISCUSSION}

Our results demonstrate that participants played video games with the intention of regulating their emotions as a response to everyday life. Participants reported feeling relieved and less stressed after playing video games. This finding indicates that playing video games could be used as means of digital emotion regulation, as one of the significant features of digital emotion regulation tools is their all-time accessibility and availability on a personal device, e.g., smartphone [43, 47, 48]. This accessibility and the availability of different sensing modalities to detect emotions, such as frontfacing cameras [36], potentially enables smartphones to proactively provide emotion regulation tools, similar to cognition-aware recommender systems [8]. However, to continuously detect the emotional state of the user, it requires sensors that collect data even when the smartphone is not in use, e.g., wristbands, smartwatches, or glasses [40].

Another main motivation for our participants to play video games was to socialise with their friends. Participants mentioned that by playing video games they spend time with their friends and enjoy the jokes and fun that occur during the game session. These findings are in line with prior research showing that socialising is one of the main motivators to play online games Yee [49]. This might be due to the fact that friends can provide social support which decreases physiological 
responses to stressors [41]. Therefore, the social aspect of gaming allows video game players to regulate their emotions and act as interpersonal emotion regulation [50].

We have also found that playing video games can lead to experiencing both intentional and unintentional emotional experiences, and is highly dependent on the result of the game. For example, in the case of a victory, participants felt happy and inspired, while in the case of a loss, participants experienced frustration and annoyance. These results are in line with prior research that shows digital emotion regulation strategies, similar to non-digital ones, can lead to both negative [35] and positive [7] outcomes. However, we note that research has shown that feeling negative emotions does not necessarily lead to negative experiences; rather the experience of both positive and negative emotions compose people's subjective well-being [38].

Moreover, our results show that participants also mentioned they played video games without an intention of changing/regulating their emotions, but rather to experience a beautiful scenery or to spend the time. These findings indicate that although video games might potentially be used for digital emotion regulation, there are other reasons behind user's motivation to play video games [49].

In our future work we plan to run a more extensive study and investigate other criteria that we did not consider in this research, such as examining if different types of video games are used to regulate emotions. We also plan to have a wider range of participants to observe if age or gender have an effect on digital emotion regulation strategies. We further plan to study if different platforms are part of the player's emotion regulation strategies. In our current study we noticed that gamers who play video games on a regular basis tend to do it for communicating with friends and teammates, which often results in emotion regulation. However, those who do not play so frequently, tend to use video games intentionally to regulate their emotions. Nevertheless, our sample size was not sufficient to support or disprove this observation. Hence, it remains open for investigation in future work.

\section{CONCLUSION}

In this work we investigate how video games are used for emotion regulation. Our findings show that participants play video games to regulate their emotions caused by their real-world situation. Our results also show that video games are used as means of socialisation and communication with friends, and lead to experiencing both intentional and unintentional emotional changes depending on the elements of the game such as end result or game settings. We conclude that video games have strong potential to become an accessible and affordable means of digital emotion regulation; however, some research challenges still remain open. We plan to extend this work in the future to a larger participant sample to understand the effects of different video game types, participant's personal and demographic characteristics on digital emotion regulation.

\section{ACKNOWLEDGMENTS}

This work is partially funded by the ARC Discovery Project DP190102627 and NHMRC grant 1170937. Zhanna Sarsenbayeva is supported by a Doreen Thomas Postdoctoral Fellowship.

\section{REFERENCES}

[1] 2007. Individual differences in cognitive reappraisal: Experiential and physiological responses to an anger provocation. International fournal of Psychophysiology 66, 2 (2007), 116 - 124. Psychophysiology of Anger.

[2] 2015. Emotion regulation, procrastination, and watching cat videos online: Who watches Internet cats, why, and to what effect? Computers in Human Behavior 52 (2015), 168 - 176.

[3] Entertainment Software Association. 2016. Essential facts about the computer and video game industry.

[4] Lisa Feldman Barrett and James J Gross. 2001. Emotional intelligence: A process model of emotion representation and regulation. (2001). 
[5] Bruce D. Bartholow, Brad J. Bushman, and Marc A. Sestir. 2006. Chronic violent video game exposure and desensitization to violence: Behavioral and event-related brain potential data. Fournal of Experimental Social Psychology 42, 4 (2006), 532 - 539. https://doi.org/10.1016/j.jesp.2005.08.006

[6] Richard Chambers, Eleonora Gullone, and Nicholas B. Allen. 2009. Mindful emotion regulation: An integrative review. Clinical Psychology Review 29, 6 (2009), 560 - 572. https://doi.org/10.1016/j.cpr.2009.06.005

[7] Emily Collins and Anna L. Cox. 2014. Switch on to games: Can digital games aid post-work recovery? International fournal of Human-Computer Studies 72, 8 (2014), 654 - 662. https://doi.org/10.1016/j.ijhcs.2013.12.006 Designing for emotional wellbeing.

[8] Tilman Dingler, Benjamin Tag, Sabrina Lehrer, and Albrecht Schmidt. [n.d.]. Reading Scheduler: Proactive Recommendations to Help Users Cope with Their Daily Reading Volume. In Proceedings of the 17th International Conference on Mobile and Ubiquitous Multimedia (MUM 2018). ACM, New York, NY, USA, 239-244. https://doi.org/10.1145/3282894.3282917

[9] Malte Elson and Christopher J Ferguson. 2014. Twenty-five years of research on violence in digital games and aggression. European Psychologist (2014)

[10] Barbara L. Fredrickson. 2003. The Value of Positive Emotions: The emerging science of positive psychology is coming to understand why it's good to feel good. American Scientist 91, 4 (2003), 330-335. http://www.jstor.org/stable/27858244

[11] Jorge Goncalves, Pratyush Pandab, Denzil Ferreira, Mohammad Ghahramani, Guoying Zhao, and Vassilis Kostakos. 2014. Projective Testing of Diurnal Collective Emotion. Association for Computing Machinery, New York, NY, USA https://doi.org/10.1145/2632048.2636067

[12] James J. Gross. [n.d.]. Emotion regulation: Affective, cognitive, and social consequences. Psychophysiology 39, 3 ([n. d.]), 281-291. https://doi.org/10.1017/S0048577201393198

[13] James J Gross. 1998. Antecedent-and response-focused emotion regulation: divergent consequences for experience, expression, and physiology. Journal of personality and social psychology 74, 1 (1998), 224

[14] James J. Gross. 1998. The Emerging Field of Emotion Regulation: An Integrative Review. Review of General Psychology 2, 3 (1998), 271-299. https://doi.org/10.1037/1089-2680.2.3.271

[15] James J. Gross. 1999. Emotion Regulation: Past, Present, Future. Cognition and Emotion 13, 5 (1999), 551-573. https://doi.org/10.1080/026999399379186

[16] James J. Gross. 2001. Emotion Regulation in Adulthood: Timing Is Everything. Current Directions in Psychological Science 10, 6 (2001), 214-219. https://doi.org/10.1111/1467-8721.00152

[17] James J Gross. 2013. Emotion regulation: taking stock and moving forward. Emotion 13, 3 (2013), 359.

[18] James J Gross and Oliver P John. 2003. Individual differences in two emotion regulation processes: implications for affect, relationships, and well-being. Journal of personality and social psychology 85, 2 (2003), 348.

[19] James J. Gross, Gal Sheppes, and Heather L. Urry. 2011. Cognition and emotion lecture at the 2010 SPSP emotion preconference: Emotion generation and emotion regulation: A distinction we should make (Carefully). Cognition and Emotion 25, 5 (2011), 765-781. https://doi.org/10.1080/02699931.2011.555753

[20] Scott H. Hemenover and Nicholas D. Bowman. 2018. Video games, emotion, and emotion regulation: expanding the scope. Annals of the International Communication Association 42, 2 (2018), 125-143.

[21] Steven J. Kirsh, Jeffrey R. W. Mounts, and Paul V. Olczak. 2006. Violent Media Consumption and the Recognition of Dynamic Facial Expressions. Journal of Interpersonal Violence 21, 5 (2006), 571-584. https://doi.org/10.1177/ 0886260506286840 PMID: 16574633.

[22] Laura D. Kubzansky, Nansook Park, Christopher Peterson, Pantel Vokonas, and David Sparrow. 2011. Healthy Psychological Functioning and Incident Coronary Heart Disease: The Importance of Self-regulation. Archives of General Psychiatry 68, 4 (04 2011), 400-408. https://doi.org/10.1001/archgenpsychiatry.2011.23

[23] Reed Larson, Bernard Hecker, and Julie Norem. 1985. Students' experience with research projects: Pains, enjoyment and success. The High School fournal 69, 1 (1985), 61-69.

[24] Thomas R. Lynch, Jennifer Q. Morse, Tamar Mendelson, and Clive J. Robins. 2003. Dialectical Behavior Therapy for Depressed Older Adults: A Randomized Pilot Study. The American fournal of Geriatric Psychiatry 11, 1 (2003), 33 - 45 https://doi.org/10.1097/00019442-200301000-00006

[25] Thomas R. Lynch, William T. Trost, Nicholas Salsman, and Marsha M. Linehan. 2007. Dialectical Behavior Therapy for Borderline Personality Disorder. Annual Review of Clinical Psychology 3, 1 (2007), 181-205. https://doi.org/10.1146/ annurev.clinpsy.2.022305.095229 PMID: 17716053.

[26] Abhinav Mehrotra, Fani Tsapeli, Robert Hendley, and Mirco Musolesi. 2017. MyTraces: Investigating Correlation and Causation between Users' Emotional States and Mobile Phone Interaction. Proc. ACM Interact. Mob. Wearable Ubiquitous Technol. 1, 3, Article 83 (Sept. 2017), 21 pages. https://doi.org/10.1145/3130948

[27] Janelle Nimer and Brad Lundahl. 2007. Animal-Assisted Therapy: A Meta-Analysis. Anthrozoös 20, 3 (2007), $225-238$.

[28] Radha O’Meara. 2014. Do cats know they rule YouTube? Surveillance and the pleasures of cat videos. M/C Fournal 17, 2 (2014). 
[29] Reinhard Pekrun, Thomas Götz, Wolfram Titz, and Raymond P. Perry. 2002. Positive emotions in education. In Beyond coping: Meeting goals, visions, and challenges, Erica Frydenberg (Ed.). Oxford University Press, Oxford, 149-173.

[30] Andrew K Przybylski, Edward L Deci, C Scott Rigby, and Richard M Ryan. 2014. Competence-impeding electronic games and players' aggressive feelings, thoughts, and behaviors. Journal of personality and social psychology 106, 3 (2014), 441.

[31] Niklas Ravaja, Timo Saari, Mikko Salminen, Jari Laarni, and Kari Kallinen. 2006. Phasic Emotional Reactions to Video Game Events: A Psychophysiological Investigation. Media Psychology 8, 4 (2006), 343-367.

[32] Byron Reeves and Clifford Nass. 1996. How people treat computers, television, and new media like real people and places.

[33] Leonard Reinecke, Tilo Hartmann, and Allison Eden. 2014. The Guilty Couch Potato: The Role of Ego Depletion in Reducing Recovery Through Media Use. Journal of Communication 64, 4 (06 2014), 569-589.

[34] Jane M. Richards and James J. Gross. 1999. Composure at Any Cost? The Cognitive Consequences of Emotion Suppression. Personality and Social Psychology Bulletin 25, 8 (1999), 1033-1044. https://doi.org/10.1177/01461672992511010

[35] Dmitri Rozgonjuk and Jon D Elhai. 2019. Emotion regulation in relation to smartphone use: Process smartphone use mediates the association between expressive suppression and problematic smartphone use. Current Psychology (2019), $1-10$.

[36] Zhanna Sarsenbayeva, Gabriele Marini, Niels van Berkel, Chu Luo, Weiwei Jiang, Kangning Yang, Greg Wadley, Tilman Dingler, Vassilis Kostakos, and Jorge Goncalves. 2020. Does Smartphone Use Drive Our Emotions or Vice Versa? A Causal Analysis. Association for Computing Machinery, New York, NY, USA. https://doi.org/10.1145/3313831.3376163

[37] Zhanna Sarsenbayeva, Niels van Berkel, Danula Hettiachchi, Weiwei Jiang, Tilman Dingler, Eduardo Velloso, Vassilis Kostakos, and Jorge Goncalves. 2019. Measuring the effects of stress on mobile interaction. Proceedings of the ACM on Interactive, Mobile, Wearable and Ubiquitous Technologies 3, 1 (2019), 1-18.

[38] Ulrich Schimmack, Shigehiro Oishi, and Ed Diener. 2005. Individualism: A valid and important dimension of cultural differences between nations. Personality and Social Psychology Review 9, 1 (2005), 17-31.

[39] Jerry Suls and James Bunde. 2005. Anger, anxiety, and depression as risk factors for cardiovascular disease: the problems and implications of overlapping affective dispositions. Psychological bulletin 131, 2 (2005), 260.

[40] Benjamin Tag, Andrew W Vargo, Aman Gupta, George Chernyshov, Kai Kunze, and Tilman Dingler. [n.d.]. Continuous Alertness Assessments: Using EOG Glasses to Unobtrusively Monitor Fatigue Levels In-The-Wild. In Proceedings of the 2019 CHI Conference on Human Factors in Computing Systems (CHI '19). ACM, New York, NY, USA, 464:1--464:12. https://doi.org/10.1145/3290605.3300694

[41] Bert N Uchino, John T Cacioppo, and Janice K Kiecolt-Glaser. 1996. The relationship between social support and physiological processes: a review with emphasis on underlying mechanisms and implications for health. Psychological bulletin 119, 3 (1996), 488.

[42] Niels van Berkel, Simon Dennis, Michael Zyphur, Jinjing Li, Andrew Heathcote, and Vassilis Kostakos. 2020. Modeling interaction as a complex system. Human-Computer Interaction 0, 0 (2020), 1-27. https://doi.org/10.1080/07370024 2020.1715221

[43] Niels van Berkel, Jorge Goncalves, Simo Hosio, Zhanna Sarsenbayeva, Eduardo Velloso, and Vassilis Kostakos. 2020. Overcoming compliance bias in self-report studies: A cross-study analysis. International fournal of Human-Computer Studies 134 (2020), 1 - 12. https://doi.org/10.1016/j.ijhcs.2019.10.003

[44] Daniela Villani, Claudia Carissoli, Stefano Triberti, Antonella Marchetti, Gabriella Gilli, and Giuseppe Riva. 2018 Videogames for emotion regulation: a systematic review. Games for health journal 7, 2 (2018), 85-99.

[45] Aku Visuri, Zhanna Sarsenbayeva, Jorge Goncalves, Evangelos Karapanos, and Simon Jones. 2016. Impact of Mood Changes on Application Selection. In Proceedings of the 2016 ACM International foint Conference on Pervasive and Ubiquitous Computing: Adjunct (Heidelberg, Germany) (UbiComp '16). Association for Computing Machinery, New York, NY, USA, 535-540. https://doi.org/10.1145/2968219.2968317

[46] Greg Wadley, Amanda Krause, Jiahui Liang, Zihe Wang, and Tuck Wah Leong. 2019. Use of Music Streaming Platforms for Emotion Regulation by International Students. In Proceedings of the 31st Australian Conference on HumanComputer-Interaction (Fremantle, WA, Australia) (OZCHI'19). Association for Computing Machinery, New York, NY, USA, 337-341.

[47] Greg Wadley, Wally Smith, Peter Koval, and James J. Gross. 2020. Digital Emotion Regulation. Current Directions in Psychological Science 0, 0 (2020), 0963721420920592. https://doi.org/10.1177/0963721420920592

[48] Kangning Yang, Chaofan Wang, Zhanna Sarsenbayeva, Benjamin Tag, Tilman Dingler, Greg Wadley, and Jorge Goncalves. 2020. Benchmarking commercial emotion detection systems using realistic distortions of facial image datasets. The Visual Computer (2020). https://doi.org/10.1007/s00371-020-01881-x

[49] Nick Yee. 2006. Motivations for Play in Online Games. CyberPsychology \& Behavior 9, 6 (2006), 772-775. https: //doi.org/10.1089/cpb.2006.9.772 PMID: 17201605.

[50] Jamil Zaki and W Craig Williams. 2013. Interpersonal emotion regulation. Emotion 13, 5 (2013), 803. 


\section{University Library}

\section{- M M N E R VA A gateway to Melbourne's research publications}

Minerva Access is the Institutional Repository of The University of Melbourne

Author/s:

Sarsenbayeva, Z;Tag, B;Yan, S;Kostakos, V;Goncalves, J

Title:

Using Video Games to Regulate Emotions

Date:

2020-12-02

Citation:

Sarsenbayeva, Z., Tag, B., Yan, S., Kostakos, V. \& Goncalves, J. (2020). Using Video Games to Regulate Emotions. ACM International Conference Proceeding Series, pp.755-759. ACM. https://doi.org/10.1145/3441000.3441035.

Persistent Link:

http://hdl.handle.net/11343/272722 